\title{
Semidefinite programming bounds for spherical codes
}

\author{
Christine Bachoc \\ IMB, Université Bordeaux I \\ 351, cours de la Libération \\ 33405 Talence France \\ bachoc@math.u-bordeaux1.fr
}

\author{
Frank Vallentin \\ Centrum voor Wiskunde en Informatica (CWI) \\ Kruislaan 413, 1098 SJ \\ Amsterdam The Netherlands \\ f.vallentin@cwi.nl
}

\begin{abstract}
This paper develops a new method to obtain upper bounds for spherical codes, based on semidefinite programming. With this method we improve the previous bounds for the kissing number in several dimensions, as well as other classical problems like Tammes' problem.
\end{abstract}

\section{INTRODUCTION}

It is a fundamental problem in coding theory to give upper bounds for the cardinality of a code in a metric space $(X, d)$ with given minimal distance. In the seventies, P. Delsarte developed a method initially aimed at bounding codes over finite fields (see [1]), that yields such an upper bound as a solution of a linear program. We shall refer to this method as the LP method; it was successfully generalized to the twopoint homogeneous spaces (see [2] for the spherical codes, [3], and the survey [4, Chap 9]), and to some other more general spaces like the non binary Johnson space (see [5]) or the Grassmannian spaces (see [6]). While the framework of association schemes is appropriate to treat the case of finite spaces ([1]), the more general point of view of harmonic analysis on symmetric spaces includes also the real spaces such as the unit sphere of the Euclidean space (see [2], [4, Chap 9]).

More recently, A. Schrijver has introduced a new method based on semidefinite programming that strengthens the LP bounds for the binary codes ([7]) and the non binary codes ([8]). His method exploits the Terwilliger algebra of the Hamming scheme.

The aim of this paper ([9]) is to develop a similar method for the spherical codes, i.e. the codes of the unit sphere $S^{n-1}$ of $\mathbb{R}^{n}$. We use the standard notation

$$
\begin{aligned}
& A(n, \theta)=\max \left\{|C|: C \subset S^{n-1}\right. \\
& \text { with } \left.c \cdot c^{\prime} \leq \cos \theta \text { for } c, c^{\prime} \in C, c \neq c^{\prime}\right\} .
\end{aligned}
$$

To be more precise, let us recall that the LP method steps on the existence of polynomials $P_{k}^{n}(t)$, satisfying the so-called positivity property:

$$
\text { for all finite } C \subset S^{n-1}, \sum_{\left(c, c^{\prime}\right) \in C^{2}} P_{k}^{n}\left(c \cdot c^{\prime}\right) \geq 0 .
$$

These polynomials arise as zonal spherical polynomials on the sphere, i.e. the zonal polynomials associated to the decompo- sition of the space of polynomial functions under the action of the orthogonal group $O\left(\mathbb{R}^{n}\right)$.

The consideration of the action restricted to a subgroup $H$ of $O\left(\mathbb{R}^{n}\right)$, chosen to be the stabilizer group of a fixed point $e \in S^{n-1}$, leads us to some symmetric matrices $S_{k}^{n}$ whose coefficients are symmetric polynomials in three variables and such that, for all finite $C \subset S^{n-1}$,

$$
\sum_{\left(c, c^{\prime}, c^{\prime \prime}\right) \in C^{3}} S_{k}^{n}\left(c \cdot c^{\prime}, c \cdot c^{\prime \prime}, c^{\prime} \cdot c^{\prime \prime}\right) \succeq 0
$$

where the sign $\succeq 0$ stands for: "is positive semidefinite". The reason why we obtain matrices instead of functions comes from the fact that, in the decomposition of the space of polynomial functions on the sphere under the action of $H$, multiplicities greater than 1 occur. In fact these multiplicities are exactly the sizes of the corresponding matrices. From (1) and (2) we derive a semidefinite program (SDP for short) whose optimal solution gives an upper bound for $A(n, \theta)$ and strengthens the LP bound. Computational results show that for several values of $n$ and $\theta$ this SDP method gives better upper bounds than the LP method. The case $\theta=\pi / 3$ is of special interest because the determination of $A(n, \pi / 3)$ is equivalent to the so-called kissing number problem.

In geometry, the kissing number problem asks for the maximum number $\tau_{n}$ of unit spheres that can simultaneously touch the unit sphere in $n$-dimensional Euclidean space without pairwise overlapping. The value of $\tau_{n}$ is only known for $n=$ $1,2,3,4,8,24$. While its determination for $n=1,2$ is trivial, it is not the case for other values of $n$. It is straightforward to see that

$$
\tau_{n}=A(n, \pi / 3)
$$

This problem has a long history, for which we refer to [4], [10]. Let us only recall that the values of $\tau_{8}$ and $\tau_{24}$ derive from the LP method (as well as uniqueness of the optimal codes), while for $n=3$ and $n=4$ it only gives the upper bounds $\tau_{3} \leq 13$ and $\tau_{4} \leq 25$. Very recently O.R. Musin, using a variation on the LP method, has proved $\tau_{4}=24$ (see [11], [12]; his method also reproves the known value $\tau_{3}=12$ ). Our SDP method obtains the exact values for the cases $n=3,4,8,24$, and improves the known bounds for $n \leq 10$ (see Table 5.1). 
We believe that our approach, based on group representation, can be easily adapted to treat many other spaces of interest in coding theory, including spaces that could not be treated with the classical LP method. An example of such spaces are the spherical caps, discussed in [13]. It is worth pointing out that A. Schrijver work can also be interpreted in group theoretic terms, involving the isometry group of the Hamming space $\mathbb{F}_{2}^{n}$ and the subgroup stabilizing $(0, \ldots, 0)$ which is the group of permutations of the $n$ positions.

The paper is organized as follows: Section II reviews on the LP method. Section III introduces and calculates the semidefinite zonal matrices associated to the action of $H$ and leading to the matrices $S_{k}^{n}$. Section IV defines the semidefinite program and its dual that establishes the desired bound. Section V discusses computational results.

\section{Review of the LP Method on the Unit Sphere}

We introduce the following notations. The standard inner product of the Euclidean space $\mathbb{R}^{n}$ is denoted by $x \cdot y$. The unit sphere

$$
S^{n-1}:=\left\{x \in \mathbb{R}^{n}: x \cdot x=1\right\}
$$

is homogeneous under the action of the orthogonal group $\mathrm{O}\left(\mathbb{R}^{n}\right)=\left\{O \in \mathbb{R}^{n \times n}: O^{t} O=I_{n}\right\}$, where $I_{n}$ denotes the identity matrix. It is moreover two-point homogeneous, meaning that the orbits of $\mathrm{O}\left(\mathbb{R}^{n}\right)$ on pairs of points are characterized by the value of their inner product. The space of real polynomial functions of degree at most $d$ on $S^{n-1}$ is denoted by $\mathrm{Pol}_{\leq d}\left(S^{n-1}\right)$. It is endowed with the induced action of $\mathrm{O}\left(\mathbb{R}^{n}\right)$, and equipped with the standard $\mathrm{O}\left(\mathbb{R}^{n}\right)$ invariant inner product

$$
(f, g)=\frac{1}{\omega_{n}} \int_{S^{n-1}} f(x) g(x) d \omega_{n}(x),
$$

where $\omega_{n}=\frac{2 \pi^{n / 2}}{\Gamma(n / 2)}$ is the surface area of $S^{n-1}$ for the standard measure $d \omega_{n}$. It is a well-known fact (see e.g. [14, Ch. 9.2]) that under the action of $\mathrm{O}\left(\mathbb{R}^{n}\right)$

$$
\mathrm{Pol}_{\leq d}\left(S^{n-1}\right)=H_{0}^{n} \perp H_{1}^{n} \perp \ldots \perp H_{d}^{n},
$$

where $H_{k}^{n}$ is isomorphic to the $\mathrm{O}\left(\mathbb{R}^{n}\right)$-irreducible space

$$
\begin{gathered}
\operatorname{Harm}_{k}^{n}=\left\{f \in \mathbb{R}\left[x_{1}, \ldots, x_{n}\right]: f\right. \text { homogeneous, } \\
\left.\operatorname{deg} f=k, \sum_{i=1}^{n} \frac{\partial^{2}}{\partial x_{i}^{2}} f=0\right\}
\end{gathered}
$$

of harmonic polynomials in $n$ variables which are homogeneous and have degree $k$. We set $h_{k}^{n}:=\operatorname{dim}\left(\operatorname{Harm}_{k}^{n}\right)=$ $\left(\begin{array}{c}n+k-1 \\ n-1\end{array}\right)-\left(\begin{array}{c}n+k-3 \\ n-1\end{array}\right)$.

A certain family of orthogonal polynomials is associated to the unit sphere. They will be denoted by $P_{k}^{n}$, with the convention that $P_{k}^{n}$ has degree $k$ and is normalized by $P_{k}^{n}(1)=1$. For $n \geq 3$ these polynomials are up to multiplicative constants Gegenbauer polynomials $C_{k}^{\lambda}$ with parameter $\lambda=n / 2-1$. So they are given by $P_{k}^{n}(t)=C_{k}^{n / 2-1}(t) / C_{k}^{n / 2-1}(1)$, and the
Gegenbauer polynomials $C_{k}^{\lambda}$ can be inductively defined by $C_{0}^{\lambda}(t)=1, C_{1}^{\lambda}(t)=2 \lambda t$, and, for $k \geq 2$,

$$
k C_{k}^{\lambda}(t)=2(k+\lambda-1) t C_{k-1}^{\lambda}(t)-(k+2 \lambda-2) C_{k-2}^{\lambda}(t)
$$

They are orthogonal with respect to the weight function $(1-$ $\left.t^{2}\right)^{\lambda-1 / 2}$ on the interval $[-1,1]$.

The polynomials $P_{k}^{n}(t)$ are related to the decomposition (3) by the so-called addition formula (see e.g. [15, Ch. 9.6]): for any orthonormal basis $\left(e_{1}, \ldots, e_{h_{k}^{n}}\right)$ of $H_{k}^{n}$ and for any pair of points $x, y \in S^{n-1}$ we have

$$
P_{k}^{n}(x \cdot y)=\frac{1}{h_{k}^{n}} \sum_{i=1}^{h_{k}^{n}} e_{i}(x) e_{i}(y) .
$$

From the addition formula (4), the positivity property (1) is straightforward.

Now we introduce the unknowns of the LP to be considered. For a spherical code $C$ we define the two point distance distribution

$$
x(u):=\frac{1}{|C|}\left|\left\{\left(c, c^{\prime}\right) \in C^{2}: c \cdot c^{\prime}=u\right\}\right|,
$$

where $u \in[-1,1]$. Clearly, only a finite number of $x(u)$ 's are not equal to zero, and the positivity property can be rewritten as a linear inequality in the $x(u)$ 's:

$$
\sum_{u \in[-1,1]} x(u) P_{k}^{n}(u) \geq 0 .
$$

Moreover, the number of elements of $C$ is given by $|C|=$ $\sum_{u \in[-1,1]} x(u)$. Noticing the obvious conditions $x(1)=1$, $x(u) \geq 0$, and $x(u)=0$ for $\cos \theta<u<1$ if the minimal angular distance of $C$ is $\theta$, we are led to consider the following linear program

$$
\begin{aligned}
& \max \left\{1+\sum_{u \in[-1, \cos \theta]} x(u):\right. \\
& x(u)=0 \text { for almost all } u \in[-1, \cos \theta], \\
& x(u) \geq 0 \text { for all } u \in[-1, \cos \theta], \\
& \left.1+\sum_{u \in[-1, \cos \theta]} x(u) P_{k}^{n}(u) \geq 0 \text { for all } k \geq 1\right\},
\end{aligned}
$$

whose optimal solution gives an upper bound for $A(n, \theta)$. The dual linear problem is

$$
\begin{aligned}
& \min \left\{1+\sum_{k \geq 1} f_{k}:\right. \\
& f_{k}=0 \text { for almost all } k \geq 1, \\
& f_{k} \geq 0 \text { for all } k \geq 1, \\
& \left.\sum_{k \geq 1} f_{k} P_{k}^{n}(u) \leq-1 \text { for all } u \in[-1, \cos \theta]\right\} .
\end{aligned}
$$

By the duality theorem (cf. [16]) any feasible solution of (7) gives an upper bound for the optimal solution of (6). The dual linear program can be restated in the following way involving polynomials:

Theorem 2.1: (see e.g. [2, Th. 4.3], [4, Ch. 9], [17]) Let $F(t)=\sum_{k=0}^{d} f_{k} P_{k}^{n}(t)$ be a polynomial of degree at most $d$ in $\mathbb{R}[t]$. If

(a) $f_{k} \geq 0$ for all $k \geq 1$ and $f_{0}>0$ and, 
then,

(b) $F(u) \leq 0$ for all $u \in[-1, \cos \theta]$,

$$
A(n, \theta) \leq \frac{F(1)}{f_{0}} .
$$

\section{SEMidefinite Zonal MATRICES}

Now we fix a point $e \in S^{n-1}$, and let $H:=\operatorname{Stab}\left(\mathrm{O}\left(\mathbb{R}^{n}\right), e\right)$ be the stabilizer of $e$ in $\mathrm{O}\left(\mathbb{R}^{n}\right)$. Obviously, $H \simeq \mathrm{O}\left(\mathbb{R}^{n-1}\right)$ since $\mathrm{O}\left(\mathbb{R}^{n-1}\right)$ can be identified with the orthogonal group of the orthogonal complement of $\mathbb{R} e$.

It is a classical result (see e.g. [14, Ch. 9.2]) that for the restricted action to $H$ the decomposition of $\operatorname{Harm}_{k}^{n}$ into $H$ irreducible subspaces is given by:

$$
\operatorname{Harm}_{k}^{n} \simeq \bigoplus_{i=0}^{k} \operatorname{Harm}_{i}^{n-1} .
$$

Hence, each of the $H_{k}^{n}$ in (3) decomposes likewise:

$$
H_{k}^{n}=H_{0, k}^{n-1} \perp H_{1, k}^{n-1} \perp \ldots \perp H_{k, k}^{n-1},
$$

where $H_{i, k}^{n-1} \simeq \operatorname{Harm}_{i}^{n-1}$.

We summarize the situation in the following picture.

$$
\begin{array}{cccccccc}
\operatorname{Pol}_{\leq d}\left(S^{n-1}\right) & & & & & & & \\
= & H_{0}^{n} & \perp & H_{1}^{n} & \perp & \ldots & \perp & H_{d}^{n} \\
= & H_{0,0}^{n-1} & \perp & H_{0,1}^{n-1} & \perp & \ldots & \perp & H_{0, d}^{n-1} \\
& & \perp & H_{1,1}^{n-1} & \perp & \ldots & \perp & H_{1, d}^{n-1} \\
& & & & & \ldots & \ldots & \ldots \\
& & & & & & \perp & H_{d, d}^{n-1}
\end{array}
$$

The isotypic components of the $H$-decomposition of the space $\mathrm{Pol}_{\leq d}\left(S^{n-1}\right)$ are the subspaces $\mathcal{I}_{k}$, for $k=0, \ldots, d$ :

$$
\mathcal{I}_{k}:=H_{k, k}^{n-1} \perp \ldots \perp H_{k, d}^{n-1} \simeq(d-k+1) \operatorname{Harm}_{k}^{n-1} .
$$

Now we show how to associate to each $\mathcal{I}_{k}$ a "zonal matrix" in view of an analogue of the addition formula (4).

Theorem 3.1: Let $\mathcal{I}=R_{0} \perp R_{1} \perp \ldots \perp R_{m} \simeq(m+1) R$ be an isotypic component of $\operatorname{Pol}_{\leq d}\left(S^{n-1}\right)$ under the action of $H$, with $R$ an $H$-irreducible space of dimension $h$. Let $\left(e_{0,1}, \ldots, e_{0, h}\right)$ be an orthonormal basis of $R_{0}$ and let $\phi_{i}$ : $R_{0} \rightarrow R_{i}$ be $H$-isomorphisms preserving the inner product on $\operatorname{Pol}_{\leq d}\left(S^{n-1}\right)$. Let $e_{i, j}=\phi_{i}\left(e_{0, j}\right)$, so that $\left(e_{i, 1}, \ldots, e_{i, h}\right)$ is an orthonormal basis of $R_{i}$. Define

$$
\begin{aligned}
E(x) & =\left(\frac{1}{\sqrt{h}} e_{i, j}(x)\right)_{\substack{0 \leq i \leq m \\
1 \leq j \leq h}} \\
& =\frac{1}{\sqrt{h}}\left(\begin{array}{ccc}
e_{0,1}(x) & \ldots & e_{0, h}(x) \\
\vdots & & \vdots \\
e_{m, 1}(x) & \ldots & e_{m, h}(x)
\end{array}\right),
\end{aligned}
$$

and

$$
Z(x, y):=E(x) E(y)^{t} \in \mathbb{R}^{(m+1) \times(m+1)} .
$$

Then the following properties hold for the matrix $Z$ :

(a) $Z(x, y)$ does not depend on the choice of the orthonormal basis of $R_{0}$. (b) The change of $\phi_{i}$ to $-\phi_{i}$ for some $i$ or the choice of another decomposition of $\mathcal{I}$ as a sum of $m+1$ orthogonal $H$ submodules changes $Z(x, y)$ to some $O Z(x, y) O^{t}$ with $O \in$ $O\left(\mathbb{R}^{m+1}\right)$.

(c) For all $g \in H, Z(g(x), g(y))=Z(x, y)$.

(d) (Matrix-type positivity property)

$$
\text { For all finite } C \subset S^{n-1}, \sum_{\left(c, c^{\prime}\right) \in C^{2}} Z\left(c, c^{\prime}\right) \succeq 0 .
$$

Proof: Omitted, see [9]

The orbits of $H$ on pairs of points on the unit sphere $x, y \in S^{n-1}$ are characterized by the values of the three inner products $e \cdot x, e \cdot y$, and $x \cdot y$. Property (c) of Theorem 3.1 implies that each coefficient $Z_{i, j}(x, y)$ of $Z(x, y)$ can be expressed as a polynomial in the three variables $u=e \cdot x, v=e \cdot y$, and $t=x \cdot y$.

By $Z_{k}^{n}$, for $0 \leq k \leq d$, let us denote the matrix associated to $\mathcal{I}_{k}$ as defined above, and more precisely to the decomposition (8) of $\mathcal{I}_{k}$. Now we shall calculate the matrix $Y_{k}^{n}(u, v, t)$ with

$$
Z_{k}^{n}(x, y)=Y_{k}^{n}(e \cdot x, e \cdot y, x \cdot y) .
$$

Theorem 3.2: With the above notations, we have, for all $0 \leq i, j \leq d-k$,

$$
\left(Y_{k}^{n}\right)_{i, j}(u, v, t)=\lambda_{i, j} P_{i}^{n+2 k}(u) P_{j}^{n+2 k}(v) Q_{k}^{n-1}(u, v, t),
$$

where

$$
\begin{aligned}
Q_{k}^{n-1}(u, v, t) & :=\left(\left(1-u^{2}\right)\left(1-v^{2}\right)\right)^{k / 2} P_{k}^{n-1}(T), \\
T & =\frac{t-u v}{\sqrt{\left(1-u^{2}\right)\left(1-v^{2}\right)}}
\end{aligned}
$$

and

$$
\lambda_{i, j}=\frac{\omega_{n}}{\omega_{n-1}} \frac{\omega_{n+2 k-1}}{\omega_{n+2 k}}\left(h_{i}^{n+2 k} h_{j}^{n+2 k}\right)^{1 / 2} .
$$

Proof: Omitted, see [9].

Due to the specific choice of the unit vector $e$ defining the subgroup $H$, the coefficients of $Y_{k}^{n}$ are not symmetric polynomials. We introduce the symmetrization $S_{k}^{n}$ of $Y_{k}^{n}$ and state the announced property (2).

Corollary 3.3: For all $d \geq 0$, for all $k \geq 0$, let $Y_{k}^{n}$ be the matrix in Theorem 3.2 and let $S_{k}^{n}$ be defined by

$$
S_{k}^{n}=\frac{1}{\left|\mathrm{~S}_{3}\right|} \sum_{\sigma \in \mathrm{S}_{3}} \sigma Y_{k}^{n},
$$

where $\mathrm{S}_{3}$ denotes the group of permutations of $\{u, v, t\}$ acting on matrix coefficients in the obvious way. Then the matrices $S_{k}^{n}$ are symmetric and have symmetric polynomials as coefficients. We have, for all finite $C \subset S^{n-1}$,

$$
\sum_{\left(c, c^{\prime}\right) \in C^{2}} Y_{k}^{n}\left(e \cdot c, e \cdot c^{\prime}, c \cdot c^{\prime}\right) \succeq 0,
$$

and

$$
\sum_{\left(c, c^{\prime}, c^{\prime \prime}\right) \in C^{3}} S_{k}^{n}\left(c \cdot c^{\prime}, c \cdot c^{\prime \prime}, c^{\prime} \cdot c^{\prime \prime}\right) \succeq 0 .
$$

Proof: Omitted, see [9]. 


\section{The Semidefinite Programming Bound}

In this section we set up the announced SDP. For a spherical code $C$ we define the three points distance distribution

$$
x(u, v, t):=\frac{1}{|C|}\left|\left\{\left(c, c^{\prime}, c^{\prime \prime}\right) \in C^{3}: \begin{array}{l}
c \cdot c^{\prime}=u \\
c \cdot c^{\prime \prime}=v \\
c^{\prime} \cdot c^{\prime \prime}=t
\end{array}\right\}\right|
$$

where $u, v, t \in[-1,1]$ and the matrix

$$
\left(\begin{array}{lll}
1 & u & v \\
u & 1 & t \\
v & t & 1
\end{array}\right)
$$

being the Gram matrix of three vectors on a unit sphere, is positive semidefinite.

The last condition together with the first is equivalent to the fact that the determinant of the Gram matrix is non-negative, hence

$$
1+2 u v t-u^{2}-v^{2}-t^{2} \geq 0 .
$$

The two point distance distribution $x(u)$ as defined in Section II and the three point distance distribution $x(u, v, t)$ are related by $x(u, u, 1)=x(u)$. The three point distance distribution satisfies the following obvious properties:

$$
\begin{aligned}
& x(u, v, t) \geq 0, \\
& x(1,1,1)=1, \\
& x(\sigma(u), \sigma(v), \sigma(t))=x(u, v, t) \text { for all } \sigma \in \mathrm{S}_{3}, \\
& \sum_{u, v, t} x(u, v, t)=|C|^{2}, \\
& \sum_{u} x(u, u, 1)=|C| .
\end{aligned}
$$

Furthermore, from the positivity properties (5) and (14), we have:

$$
\begin{aligned}
& \sum_{u} x(u, u, 1) P_{k}^{n}(u) \geq 0 \text { for all } k \geq 1, \\
& \sum_{u, v, t} x(u, v, t) S_{k}^{n}(u, v, t) \succeq 0 \text { for all } k \geq 0 .
\end{aligned}
$$

If the minimal angular distance of $C$ is $\theta$, we have moreover

$$
x(u, v, t)=0 \text { whenever } u, v, t \notin[-1, \cos \theta] \cup\{1\} .
$$

To factor out the action of $\mathrm{S}_{3}$ we introduce the domains

$$
\begin{gathered}
D=\left\{(u, v, t): \begin{array}{c}
-1 \leq u \leq v \leq t \leq \cos \theta \\
1+2 u v t-u^{2}-v^{2}-t^{2} \geq 0
\end{array}\right\} \\
D_{0}=\{(u, u, 1):-1 \leq u \leq \cos \theta\}, \quad I=[-1, \cos \theta],
\end{gathered}
$$

and

$$
m(u, v, t)=\left[\mathrm{S}_{3}: \operatorname{Stab}\left(\mathrm{S}_{3},(u, v, t)\right)\right],
$$

so that, more explicitly:

$$
m(u, v, t)= \begin{cases}6 & \text { if } u \neq v \neq t \\ 3 & \text { if } u=v \neq t \text { or } u \neq v=t \text { or } u=t \neq v \\ 1 & \text { if } u=v=t\end{cases}
$$

Moreover, we introduce the notation $z:=(u, v, t)$. From the discussion above, a solution to the following semidefinite program in the variables $x^{\prime}(z)=m(z) x(z)$ is an upper bound for $A(n, \theta)$ :

$$
\begin{aligned}
& \max \left\{1+\frac{1}{3} \sum_{z \in D_{0}} x^{\prime}(z):\right. \\
& x^{\prime}(z)=0 \text { for almost all } z \in D \cup D_{0}, \\
& x^{\prime}(z) \geq 0 \text { for all } z \in D \cup D_{0}, \\
& \left(\begin{array}{ll}
1 & 0 \\
0 & 0
\end{array}\right)+\frac{1}{3} \sum_{u \in I} x^{\prime}(z)\left(\begin{array}{ll}
0 & 1 \\
1 & 1
\end{array}\right)+\sum_{z \in D} x^{\prime}(z)\left(\begin{array}{ll}
0 & 0 \\
0 & 1
\end{array}\right) \succeq 0, \\
& 3+\sum_{z \in D_{0}} x^{\prime}(z) P_{k}^{n}(z[1]) \geq 0 \text { for all } k \geq 1, \\
& \left.S_{k}^{n}(1,1,1)+\sum_{z \in D \cup D_{0}} x^{\prime}(z) S_{k}^{n}(z) \succeq 0 \text { for all } k \geq 0\right\} .
\end{aligned}
$$

Just like in the LP method, the main problem with the above SDP, is that the unknowns $x(z)$ are indexed by a continuous domain of $\mathbb{R}^{3}$. We cannot exploit the information that only a finite number of them are not equal to zero, because we don't know to which values of $z$ they correspond. In order to go round this difficulty, we apply the duality theorem. In the following theorem we give the SDP dual to the above one. We use the standard notation for the inner product of symmetric matrices: $\langle A, B\rangle=\operatorname{Trace}(A B)$. Furthermore we apply the simplification $S_{k}^{n}(1,1,1)=0$ for $k \geq 1$.

Theorem 4.1: Any feasible solution of the following semidefinite problem gives an upper bound on $A(n, \theta)$ :

$$
\begin{aligned}
& \min \left\{1+\sum_{k \geq 1} a_{k}+b_{11}+\left\langle F_{0}, S_{0}^{n}(1,1,1)\right\rangle:\right. \\
& a_{k}=0 \text { for almost all } k \geq 1, \\
& a_{k} \geq 0 \text { for all } k \geq 1, \\
& \left(\begin{array}{ll}
b_{11} & b_{12} \\
b_{12} & b_{22}
\end{array}\right) \succeq 0, \\
& F_{k}=0 \text { for almost all } k \geq 0, \\
& F_{k} \succeq 0 \text { for all } k \geq 0, \\
& \sum_{k \geq 1} a_{k} P_{k}^{n}(z[1])+2 b_{12}+b_{22}+3 \sum_{k \geq 0}\left\langle F_{k}, S_{k}^{n}(z)\right\rangle \leq-1, \\
& \left.b_{22}+\sum_{k \geq 0}\left\langle F_{k}, S_{k}^{n}(z)\right\rangle \leq 0\right\},
\end{aligned}
$$

where last inequality holds for all $z \in D$ and the second but last inequality holds for all $z \in D_{0}$.

\section{Computational Results}

One possible strategy to derive explicit and rigorous upper bounds for $\tau_{n}$ from Theorem 4.1 makes use of techniques from polynomial optimization introduced e.g. in [18] and [19], [20]. Due to space considerations, we skip detailed explanations here, referring instead to [9], and only give numerical results.

The values of the last column were found by solving the above semidefinite program for the value $d=10$. The values of the third column were obtained by Odlyzko and Sloane by Theorem 2.1 using the value $d=30$. They pointed 


\begin{tabular}{|c|c|c|c|c|}
\hline$n$ & $\begin{array}{l}\text { best lower } \\
\text { bound known }\end{array}$ & $\begin{array}{l}\text { best upper bound } \\
\text { previously known }\end{array}$ & $\begin{array}{l}\text { LP } \\
\text { method }\end{array}$ & $\begin{array}{l}\text { SDP } \\
\text { method }\end{array}$ \\
\hline 3 & 12 & $\begin{array}{ll}12 & \text { ([21]) }\end{array}$ & 13 & 12 \\
\hline 4 & 24 & $24 \quad([11])$ & 25 & 24 \\
\hline 5 & 40 & 46 ([17]) & 46 & 45 \\
\hline 6 & 72 & 82 ([17]) & 82 & 78 \\
\hline 7 & 126 & 140 ([17]) & 140 & 135 \\
\hline 8 & 240 & 240 ([17], [18]) & 240 & 240 \\
\hline 9 & 306 & 379 ([22]) & 380 & 366 \\
\hline 10 & 500 & 594 ([23]) & 595 & 567 \\
\hline
\end{tabular}

Table 5.1. Bounds on $\tau_{n}$.

out that even $d=11$ would suffice for $n \leq 10$. Our calculations were performed by the program csdp (Version 5.0) of B. Borchers ([24]) which is available on the Internet (http://infohost.nmt.edu/ $\sim$ borchers/csdp.html). After solving the SDP with csdp we checked independently whether the solution satisfies the desired constraints. This can be done using rational arithmetic only. So our computations give rigorous proofs of the stated upper bounds. Due to numerical instabilities we were not able to perform this calculation for larger $n$ and/or larger $d$.

For the lower bounds in the first column we refer to the Catalogue of Lattices of G. Nebe and N.J.A. Sloane (http://www.research.att.com/ njas/lattices/kiss.html).

We also computed upper bounds for $A\left(n, \cos ^{-1} 1 / 3\right)$. Hereby we improved several entries of the Table 9.2 of [4] where all best upper bounds previously known were obtained by the LP method. We give our results in Table 5.2. Again we used the value $d=10$ to obtain the last column.

\begin{tabular}{c|c|c|c}
$n$ & $\begin{array}{c}\text { best lower } \\
\text { bound known }\end{array}$ & $\begin{array}{c}\text { best upper bound } \\
\text { previously known }\end{array}$ & $\begin{array}{c}\text { SDP } \\
\text { method }\end{array}$ \\
\hline 3 & 9 & 9 & 9 \\
4 & 14 & 15 & 15 \\
5 & 20 & 24 & 23 \\
6 & 32 & 37 & 35 \\
7 & 56 & 56 & 56 \\
8 & 64 & 78 & 74 \\
9 & 96 & 107 & 99 \\
10 & & 146 & 135
\end{tabular}

Table 5.2. Bounds on $A\left(n, \cos ^{-1} 1 / 3\right)$.

We were also able to improve the best known upper bounds for the so-called Tammes' problem with $N$ spheres: What is the largest minimal angle $\theta(N)$ that can be obtained by a spherical code of $S^{2}$ with cardinality $N$. Let us recall that the answer is only known for $N \leq 12$ and for $N=24$ (see [4, Chap 1]). For $N=13$, the best known lower bound is $0.997223593 \approx 57.1367031^{\circ}$ whereas the best-known upper bound is $1.02746114 \lesssim 58.8691870^{\circ}$ due to $\mathrm{K}$. Böröczky and L. Szabo [25]. We obtained $A\left(3, \cos ^{-1}(0.5225)\right) \leq 12.99$ using $d=N=10$, giving the new upper bound of $1.02101593 \lesssim 58.4999037^{\circ}$. Other values are collected in Table 5.3; the lower bounds are taken from the homepage of N.J.A. Sloane (http://www.research.att.com/ njas/packings). The upper bounds for $N \geq 14$ where established in [26].

\begin{tabular}{c|c|c|c}
$N$ & $\begin{array}{c}\text { best lower } \\
\text { bound known }\end{array}$ & $\begin{array}{c}\text { best upper bound } \\
\text { previously known }\end{array}$ & $\begin{array}{c}\text { SDP } \\
\text { method }\end{array}$ \\
\hline 13 & 57.13 & 58.87 & 58.50 \\
14 & 55.67 & 58.00 & 56.58 \\
15 & 53.65 & 55.84 & 55.03 \\
16 & 52.24 & 53.92 & 53.27 \\
17 & 51.09 & 52.11 & 51.69
\end{tabular}

Table 5.3. Bounds on $\theta(N)$ (given in degrees).

\section{REFERENCES}

[1] P. Delsarte, An algebraic approach to the association schemes of coding theory, Philips Res. Rep. Suppl. vi+97, 1973.

[2] P. Delsarte, J.M. Goethals and J.J. Seidel, "Spherical codes and designs", Geom. Dedicata vol. 6, pp. 363-388, 1977,

[3] V.I. Levenshtein, "On bounds for packing in $n$-dimensional Euclidean space", Soviet Math. Dokl. vol. 20, pp. 417-421, 1979.

[4] J.H. Conway and N.J.A. Sloane, Sphere Packings, Lattices and Groups, Springer-Verlag, 1988.

[5] M.J. Aaltonen, "A new upper bound on non binary block codes", Discrete Math., vol. 83, pp. 139-160, 1990.

[6] C. Bachoc, "Linear programming bounds for codes in Grassmannian spaces", IEEE Trans. Inform. Theory, vol. 52, pp. 2111-2125, 2006.

[7] A. Schrijver, "New code upper bounds from the Terwilliger algebra and semidefinite programming", IEEE Trans. Inform. Theory vol. 51, pp. 2859-2866, 2005.

[8] D.C. Gijswijt, A. Schrijver, and H. Tanaka, "New upper bounds for nonbinary codes", J. Combin. Theory Ser. A, vol. 13, pp. 1719-1731, 2006.

[9] C. Bachoc, F. Vallentin, "New upper bounds for kissing numbers from semidefinite programming", preprint, October 2006, arxiv:math.MG/0608426.

[10] F. Pfender, and G.M. Ziegler, "Kissing numbers, sphere packings and some unexpected proofs", Notices Amer. Math. Soc., vol. 51, pp. 873883, 2004.

[11] O.R. Musin, "The kissing number in four dimensions", preprint, April 2005, arxiv:math.MG/0309430.

[12] O.R. Musin, "The kissing problem in three dimensions", Discrete Comput. Geom. vol. 35, pp. 375-384, 2006.

[13] C. Bachoc, F. Vallentin, "Semidefinite programming, multivariate orthogonal polynomials, and codes in spherical caps", preprint, October 2006, arxiv:math.MG/0610856.

[14] N.Ja. Vilenkin, and A.U. Klimyk, Representation of Lie Groups and Special Functions, Volume 2, Kluwer Academic Publishers, 1993.

[15] G.E. Andrews, R. Askey, and R. Roy, Special functions, Cambridge University Press, 1999.

[16] R.J. Duffin, "Infinite programs", in Linear inequalities and related systems, (H.W. Kuhn and A.W. Tucker eds.), Princeton Univ. Press, pp. 157-170, 1956.

[17] A.M. Odlyzko and N.J.A. Sloane, "New bounds on the number of unit spheres that can touch a unit sphere in n dimensions", J. Combin. Theory Ser. A, vol. 26, pp. 210-214, 1979.

[18] J.B. Lasserre, "Global optimization with polynomials and the problem of moments", SIAM J. Optim., vol. 11, pp. 796-817, 2001.

[19] P.A. Parrilo, "Semidefinite programming relaxations for semialgebraic problems", Math. Program. vol. 96, pp. 293-320, 2003.

[20] M. Putinar, "Positive polynomials on compact semi-algebraic sets", Ind. Univ. Math. J., vol. 42, pp. 969-984, 1993.

[21] K. Schütte, and B.L. van der Waerden, "Das Problem der dreizehn Kugeln", Math. Ann., vol. 125, pp. 325-334, 1953.

[22] M.G. Rzhevskii and M.A. Vsemirnov, "An upper bound for the contact number in dimension 9", Russ. Math. Surv., vol. 57, pp. 1015-1016, 2002.

[23] F. Pfender, "Improved Delsarte bounds via extension of the function space", preprint, January 2005, arXiv:math.CO/0501493.

[24] B. Borchers, "CSDP, A C Library for Semidefinite Programming", Optimization Methods and Software, vol. 11, pp. 613-623, 1999.

[25] K. Böröczky, L. Szabó, "Arrangements of 13 points on a sphere", In: Discrete Geometry, A. Bezdek (ed.), Dekker 2003, pp. 111-184.

[26] K. Böröczky, L. Szabó, "Arrangements of 14, 15, 16 and 17 points on a sphere", Studi. Sci. Math. Hung., vol. 40, pp. 407-421, 2003. 